\title{
WBRT vs. APBI: an interim report of patient satisfaction and outcomes
}

\author{
Samantha M. Bitter, BS', Patricia Heffron-Cartwright, MS², Christopher Wennerstrom, MS², Jared Weatherford, MS², \\ Douglas Einstein, MD, PhD², Lovis C. Keiler, MD² \\ University of Cincinnati College of Medicine, Cincinnati, ${ }^{2}$ Kettering Medical Center, Kettering, OH, USA
}

\begin{abstract}
Purpose: To determine differences in patient's reported quality of life and self-reported breast cosmesis between whole breast radiation therapy (WBRT) and accelerated partial breast irradiation (APBI) via single and multi-lumen high-dose-rate (HDR) brachytherapy for women with early stage breast cancer.

Material and methods: Patient information was retrospectively reviewed and survey data were prospectively collected for women treated between 2004 to 2014 (APBI) and 2012 to 2014 (WBRT). Criteria for APBI treatments were ER+ (after 2010), N0 (after 2010), T $<3 \mathrm{~cm}$, and post-menopausal. All patients were given a survey with modified FACIT (Functional Assessment of Chronic Illness Therapy) breast quality of life questions to rate their amount of pain, self-consciousness, low energy, presence of lymphedema, and breast cosmesis.

Results: 242 APBI patients and 59 WBRT patients were identified. In the WBRT cohort, 34 women met departmental criteria for APBI treatment (WBRT who were APBI eligible). The FACIT survey was completed by 80 women treated with APBI (33\%; mean follow-up time of 14 months), and 26 women treated with WBRT who were APBI eligible (76\%; mean follow-up time of 26 months). During the first year post-treatment, low energy $(p=0.009)$, self-consciousness $(p=0.0004)$, and lymphedema $(p=0.0002)$ scores were significantly lower in the APBI cohort when compared to women treated with WBRT who were APBI eligible. During the second year post-treatment, women treated with APBI reported significantly better breast cosmesis $(p=0.04)$. The single-lumen balloon (score $=6.3 / 10)$ was found to be associated with worse cosmesis compared to the multi-lumen balloons (Mammosite ML and Contura; score $=8.2 / 10$; $p=0.002)$. There were no significant differences in rates of recurrence between balloons or treatments $(p>0.05)$.

Conclusions: APBI treated patients reported higher cosmetic satisfaction than patients in the matched WBRT cohort. Quality of life scores tended to improve over time. Multi-lumen catheters provided superior cosmetic results compared to single-lumen catheters.
\end{abstract}

Key words: APBI, breast cancer, Contura, Mammosite, quality, SAVI.

\section{Purpose}

Accelerated partial breast irradiation (APBI) was approved by the Food and Drug Administration (FDA) for the treatment of breast cancer in 2002 and provides patients a more attractive radiotherapy option when compared to the standard whole breast radiation therapy (WBRT). The primary benefits of APBI include a shorter total treatment time and reduced volume of breast tissue exposed to the full dose of radiation. Furthermore, the biologically effective dose (BED) of APBI treatment is comparable to that of conventional and hypofractionated whole breast techniques. For patients who meet the ASTRO (American Society for Therapeutic Oncology) Suitability Guidelines and have negative nodes and ER+ pathology (estrogen positive), studies to date have shown similar rates of local control and overall survival between APBI and WBRT [1,2]. Accelerated partial breast irradiation is the treatment of choice for approximately one in ten women receiving radiation therapy after lumpectomy, and one in six women who meet ASTRO Guidelines.

Accelerated partial breast irradiation has been found to be as cost-effective as WBRT and due to its shorter treatment schedule, can be a financially and socially more viable treatment option for patients with transportation difficulties, homes in underserved areas, and jobs that do not allow flexibility in scheduling several weeks of daily treatments $[3,4,5,6,7]$. There are several contraindications to APBI including clinical stage III or IV disease, positive surgical margins, presence of extensive intraductal component, Paget's disease of the breast, skin involvement, contralateral breast cancer, pregnancy, breast feeding, col- 
lagen vascular, genetic or other metabolic diseases with hypersensitivity to radiation [8].

For patients who meet the inclusion criteria, multiple APBI techniques are available. These include interstitial brachytherapy (high-dose-rate - HDR, pulsed-dose-rate PDR, and permanent implants), brachytherapy using single-lumen balloons (MammoSite [Hologic Inc., Bedford, MA, USA]); brachytherapy using multi-lumen balloons (MammoSite ML, Contura [SenoRx, Inc., Aliso Viejo, CA, USA]), and hybrid/strut-based brachytherapy devices (SAVI applicator [SAVI; Cianna Medical, Aliso Viejo, CA, USA]) (Figure 1). The first option, interstitial brachytherapy, requires the implantation of interstitial applicators through the lumpectomy site, which is not practiced at our hospital. The single-lumen balloon brachytherapy technique is a simpler approach that utilizes a balloon applicator consisting of a silicone balloon catheter that contains a channel for filling the balloon and a channel for introducing the radioisotope. Multi-lumen balloons permit greater dosimetric optimization. Strut based devices (SAVI) forgo an inflatable barrier between catheters and tissue allowing tissue to settle between the struts, after placement. Given the anatomic differences between struts and balloon-based devices, different dosimetric guidelines are used in determining acceptability of treatment plans. Previously published results suggest a satisfactory treatment outcome and good cosmetic results with all of these applicators $[9,10,11,12,13,14,15,16,17,18]$. However, patient assessed quality of life and cosmesis have not been compared between these different brachytherapy options.
With these considerations in mind, the aim of our study was to compare patient's reported quality of life and self-reported breast cosmesis after being treated with WBRT versus APBI. Furthermore, the differences in quality of life and cosmesis between those women treated with single-lumen, multi-lumen, and hybrid brachytherapy devices were reviewed as well.

\section{Material and methods}

\section{Subjects}

The charts of women, ages 40-87 years, who received partial breast radiation treatment for breast cancer at our hospital were retrospectively reviewed per an Institutional Review Board (IRB) approved protocol. Following an initial analysis of the data, the protocol was amended to include additional prospective data collection of women receiving both APBI and WBRT. Women treated with APBI between the years of 2002 to 2014 and women treated with WBRT between the years of 2012 to 2014 were included in this analysis. Women were treated with WBRT after completion of breast conservation surgery with lumpectomy, per the standard of care. Women who received WBRT who were APBI eligible were compared to those women who received APBI therapy for all primary and post-hoc analyses. Departmental criteria to qualify for APBI treatment was in agreement with ASTRO's Suitability Guidelines and included: post-menopausal women with estrogen receptor positive status (ER; after 2010), negative sentinel nodes (after 2010), and tumor size less than $3 \mathrm{~cm}$, qualifying them

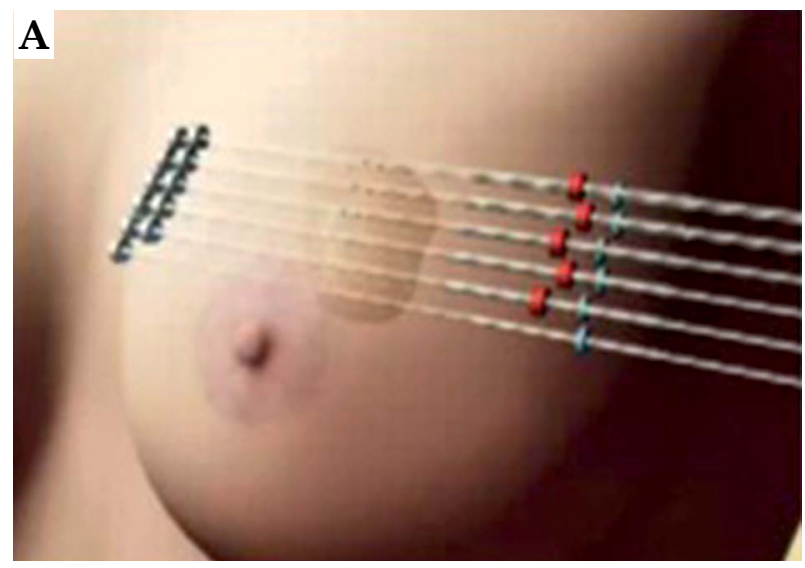

B

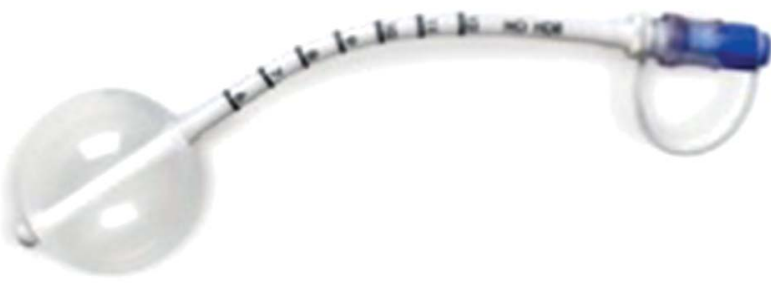

C
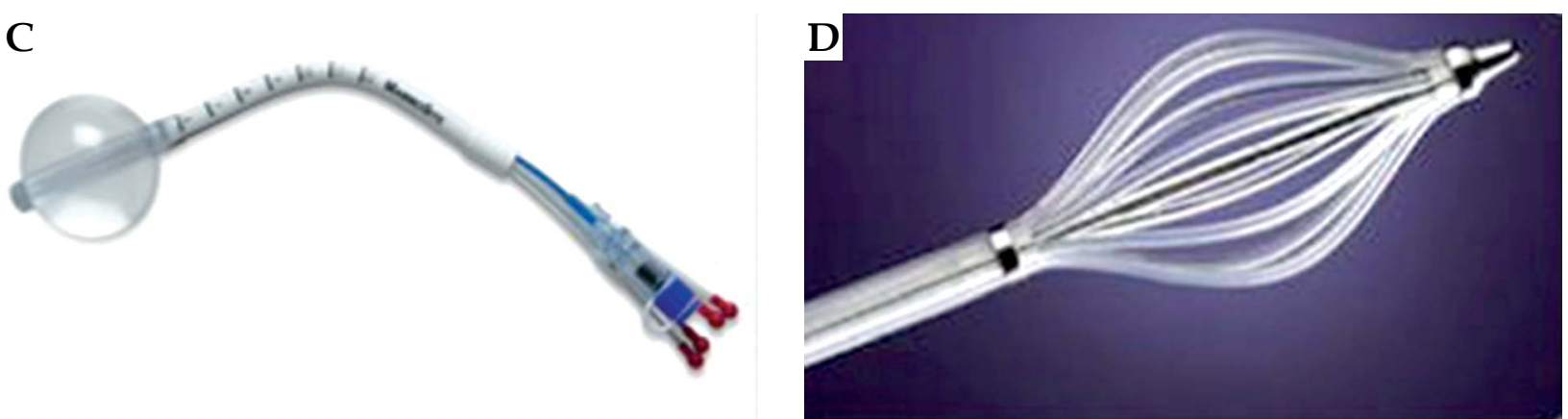

Fig. 1. Accelerated partial breast irradiation techniques. A) Interstitial brachytherapy (high-dose-rate [HDR], pulsed-doserate [PDR], and permanent implants) [28]. B) Brachytherapy using single-lumen balloons (MammoSite, Contura) [29]. C) Brachytherapy using multi-lumen balloons (MammoSite ML) [29]. D) Hybrid brachytherapy devices (SAVI applicator) [30] 
Table 1. Demographic, tumor, and treatment information of women treated with whole breast radiation therapy (WBRT), whole breast radiation therapy who were eligible for accelerated partial breast irradiation (WBRT who were accelerated partial breast irradiation [APBI] eligible), and women treated with APBI

\begin{tabular}{|c|c|c|c|}
\hline & WBRT $(n=59)$ & APBI eligible WBRT $(n=34)$ & $\mathrm{APBI}(n=242)$ \\
\hline \multicolumn{4}{|l|}{ Demographics } \\
\hline Age, mean (SD), years ${ }^{a}$ & $60(11)$ & $61(10)$ & $64(11)$ \\
\hline \multicolumn{4}{|l|}{ Tumor characteristics } \\
\hline Tumor diam, mean (SD), $\mathrm{cm}^{\mathrm{b}}$ & $2.4(2.0)$ & $1.3(0.7)$ & $1.2(0.7)$ \\
\hline \multicolumn{4}{|l|}{ Tumor stage, $n(\%)$} \\
\hline Tis & $8(14)$ & $6(18)$ & $52(21)$ \\
\hline $\mathrm{T1}$ & $27(46)$ & $24(71)$ & $171(71)$ \\
\hline $\mathrm{T} 2$ & $14(24)$ & $4(12)$ & $21(7)$ \\
\hline $\mathrm{T3}$ & $7(12)$ & $0(0)$ & $0(0)$ \\
\hline $\mathrm{T4}$ & $3(5)$ & $0(0)$ & $0(0)$ \\
\hline $\mathrm{N}+, n(\%)^{\mathrm{c}}$ & $20(34)$ & $7(21)$ & $4(2)$ \\
\hline $\mathrm{ER}+, n(\%)$ & $47(79)$ & $27(79)$ & $204(84)$ \\
\hline $\mathrm{PR}+, n(\%)$ & $46(78)$ & $27(79)$ & $196(81)$ \\
\hline \multicolumn{4}{|l|}{ Treatment information } \\
\hline \multicolumn{4}{|l|}{ Balloon type, $n(\%)$} \\
\hline Single-lumen & $\mathrm{N} / \mathrm{A}$ & $\mathrm{N} / \mathrm{A}$ & $139(58)$ \\
\hline Multi-lumen & N/A & N/A & $81(33)$ \\
\hline SAVI & N/A & N/A & $22(9)$ \\
\hline
\end{tabular}

${ }^{\mathrm{a}} p=0.007$ between groups; ${ }^{\mathrm{b}} p<0.0001$ between groups; ${ }^{c} p<0.0001$ between APBI eligible WBRT and APBI

$E R+-$ estrogen positive, $P R+-$ progesterone positive, SAVI - Strut-Adjusted Volume Implant

as Stage I or II [1]. Demographic, tumor, and treatment information on the included patients can be found in Table 1.

\section{Radiotherapy details}

Whole breast radiation therapy was defined as irradiating the whole breast with a radiation boost to the region of the tumor bed. Accelerated partial breast irradiation was defined as irradiating the region of the tumor bed alone using one of the commercially available catheter devices. After completing a CT-simulation, the patients in the WBRT group received therapy with 6-25 MV X-rays using three-dimensional conformal radiotherapy (3DCRT) to a total dose of 45-50.4 Gy. This therapy was delivered in $25-28$ fractions over a five- to six-week period, followed by a tumor bed boost with either electrons or mini-tangents for an additional 10-14.4 Gy to the tumor bed. This boost was delivered in 1.8-2 Gy fractions over five to eight days. Patients in the APBI group received $34 \mathrm{~Gy}$ in ten treatments (twice a day) for five days using an HDR afterloader.

\section{FACIT quality of life survey}

A prospective analysis of validated FACIT (Functional Assessment of Chronic Illness Therapy) breast cancer quality of life questions was performed. The survey allowed patients to rate their amount of pain, self-consciousness, low energy, and presence of lymphedema on a scale from 0 to $4(0=$ not at all, $1=$ a little bit, $2=$ somewhat, $3=$ quite a bit, $4=$ very much) and their breast cosmesis on a scale of 1-10 with a score of 10 being the best [19].

\section{Data analysis}

Statistical analyses were performed using the Statistical Analysis System (SAS Institute, Cary, NC, USA, 20022008). Student's $t$-tests and $\chi^{2} /$ Fisher's Exact Test were used to evaluate demographic characteristics between groups. Student's $t$-tests were used to compare survey scores and cosmesis scores between those women treated with APBI and women treated with WBRT who were APBI eligible for the primary analysis. Post-hoc analyses using analyses of variance (ANOVA) tests were used to examine the influence of APBI balloon type on selfreported scores. Finally, Pearson correlations were used to examine the relationship of age with self-reported scores.

\section{Results}

\section{Clinical characteristics}

From the retrospective chart analysis, 242 APBI patients and 59 WBRT patients were identified. In the WBRT 

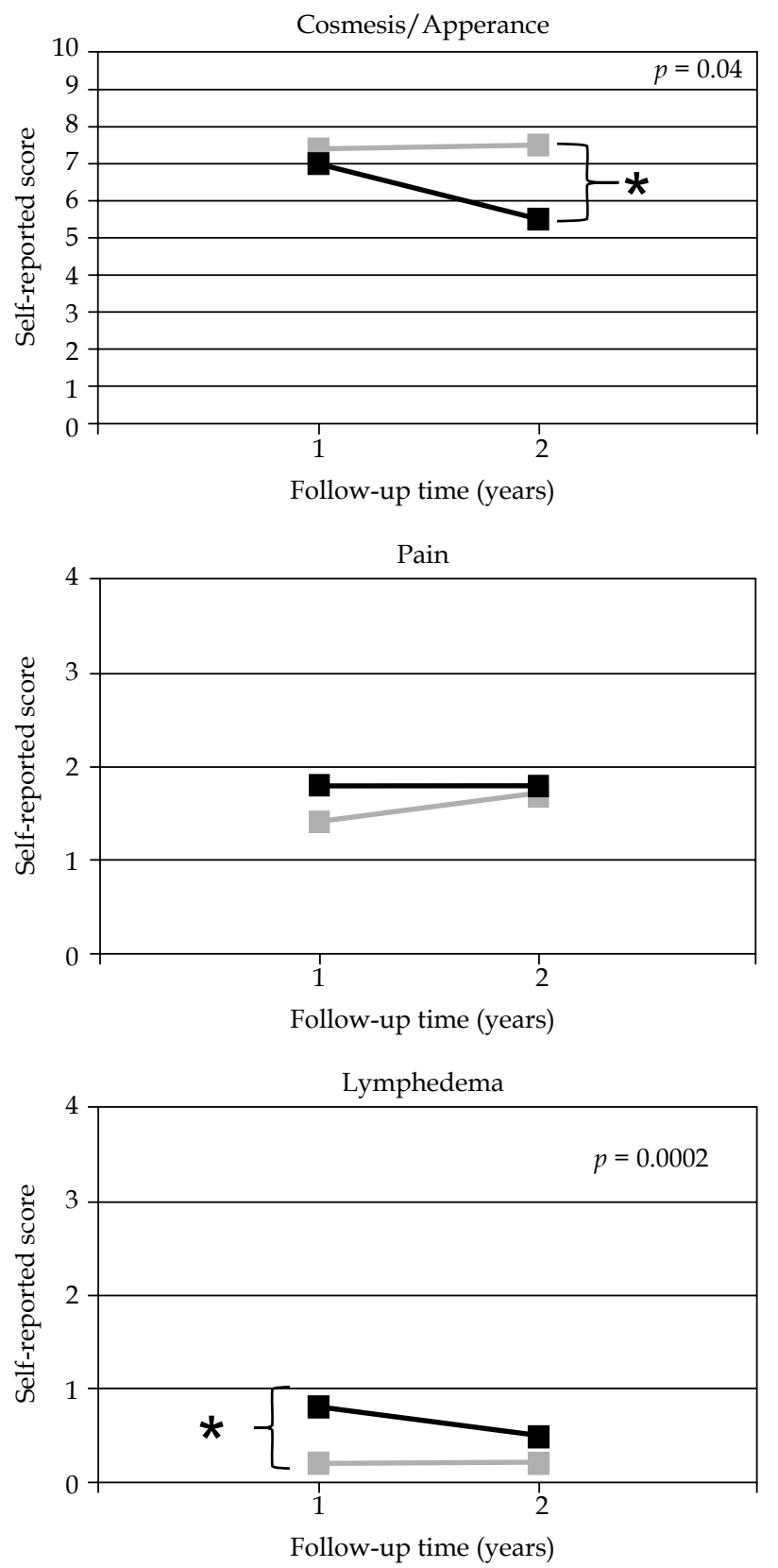

cohort, 34 women met departmental criteria for APBI treatment. Significant differences in age were observed, with the APBI treatment group being the eldest $(p=0.007)$. The FACIT survey was completed by 80 women (33\%) treated with APBI (mean follow-up time of 14 months), and 26 women $(76 \%)$ treated with WBRT who were APBI eligible (mean follow-up time of 26 months). Within the APBI cohort, 35 women $(44 \%)$ were treated with single-lumen devices, and $42(53 \%)$ women were treated with multi-lumen devices. There were no significant differences in rates of cancer recurrence between radiation techniques used $(p=0.1)$.

\section{Survey results}

During the first year post-radiation treatment, women treated with APBI reported significantly higher
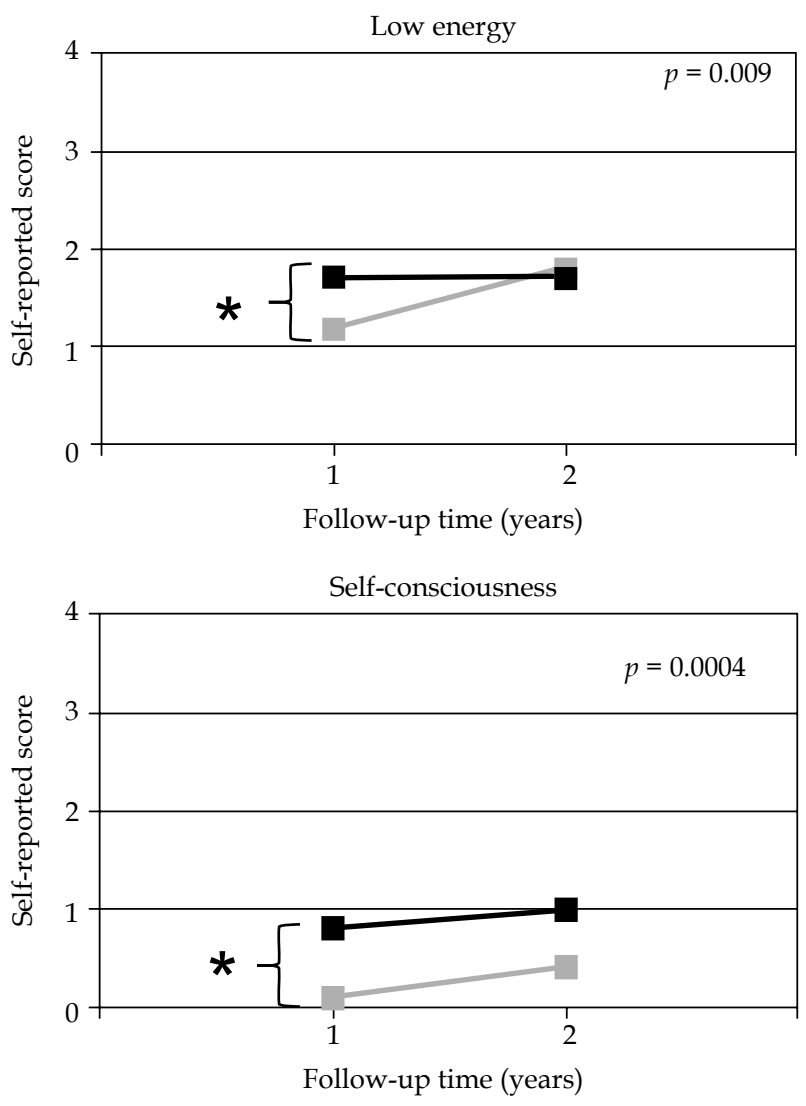

$$
\begin{aligned}
& - \text { WBRT eligible for APBI } \\
& * \text { APBI } \\
& \star p<0.05
\end{aligned}
$$

Fig. 2. Self-rated cosmetic scores and FACIT (Functional Assessment of Chronic Illness Therapy) Survey results between women treated with whole breast radiation who were eligible for accelerated partial breast irradiation (whole breast radiation therapy [WBRT] who were accelerated partial breast irradiation [APBI] eligible) and women treated with APBI

energy level scores $(p=0.009)$, and significantly lower self-consciousness $(p=0.0004)$ and lymphedema scores $(p=0.0002)$ when compared to patients treated with WBRT who were APBI eligible (Figure 2). In the second year following treatment, women treated with APBI reported significantly higher breast cosmesis $(p=0.04)$ when compared to patients treated with WBRT who were APBI eligible (Figure 2). Furthermore, in the APBI cohort, older patients reported significantly less pain ( $p=0.0008, R=-0.4)$ and self-consciousness $(p=0.02, R=-0.3)$.

\section{Accelerated partial breast irradiation balloon comparison}

The single-lumen balloon (LSmean score $=6.3 / 10$ ) was found to be associated with worse patient-graded 
breast cosmesis compared to the multi-lumen balloons (Mammosite ML and Contura; LS mean score $=8.2 / 10$; $p=0.002$; Figure 3 ). There were no significant differences in rates of cancer recurrence between the types of devices used in treatment $(p=0.7)$.

\section{Discussion}

This study suggests, in agreement with prior research, that women treated with APBI for breast cancer report better quality of life outcomes when compared to women treated with WBRT who meet ASTRO Suitability Guidelines for APBI treatment $[20,21]$. Specifically, women treated with multi-lumen catheter-based APBI are significantly happier with their breast appearance one year after treatment when compared to women treated with WBRT.

In addition to the initial difference in quality of life scores following radiation treatment with APBI, quality of life scores tended to improve over time. This phenomenon has been previously reported, and suggests overall decreased toxicity with APBI treatment, following the resolution of acute toxicity to the tumor bed $[22,23,24]$.

Furthermore, younger patients had worse self-reported cosmetic outcomes in the APBI cohort. A possible reason for this finding is initial higher scores of breast cosmesis in this group, prior to their breast surgery and radiation allowing for a greater potential difference in their breast appearance following treatment, thus presenting as lower self-rated cosmesis scores.

Finally, multi-lumen devices were found to provide superior patient rated cosmetic outcomes when compared to single-lumen catheters. The multi-lumen devices included the MammoSite ML balloon, the Contura balloon, and the SAVI device. Consistent with findings from other studies, these devices allow for dosimetric optimization to minimize toxicity to the surrounding chest wall, skin, and breast tissue $[25,26,27]$.

There are several limitations that should be considered when interpreting the results of our study. First, the sample size was small due to the limited number of patients treated at our community hospital and their charts were retrospectively reviewed. This also affected our ability to match our participants for demographic characteristics. Second, the short follow-up time for the WBRT cohort limited the number of long term comparisons that could be made between the groups, and as a result of the APBI guidelines, the WBRT cohort included a greater number of patients with advanced disease. Third, we did not treat any patients with Linac or interstitial catheter brachytherapy based APBI. Next, patients were selected for radiation therapy largely before initial consult with radiation oncology. Additionally, differences in patient perceptions of survey options may have limited their later satisfaction. Likewise, differences in surgical approach taken with WBRT and APBI groups cannot be quantified. Furthermore, there may have been a surgeon or patient selection bias with treatment decision regarding WBRT vs. APBI. Lastly, multi-lumen devices replaced single-lumen devices in our practice in entirety; therefore, differences in those approaches should be without bias. However, patient selection for strut ver-

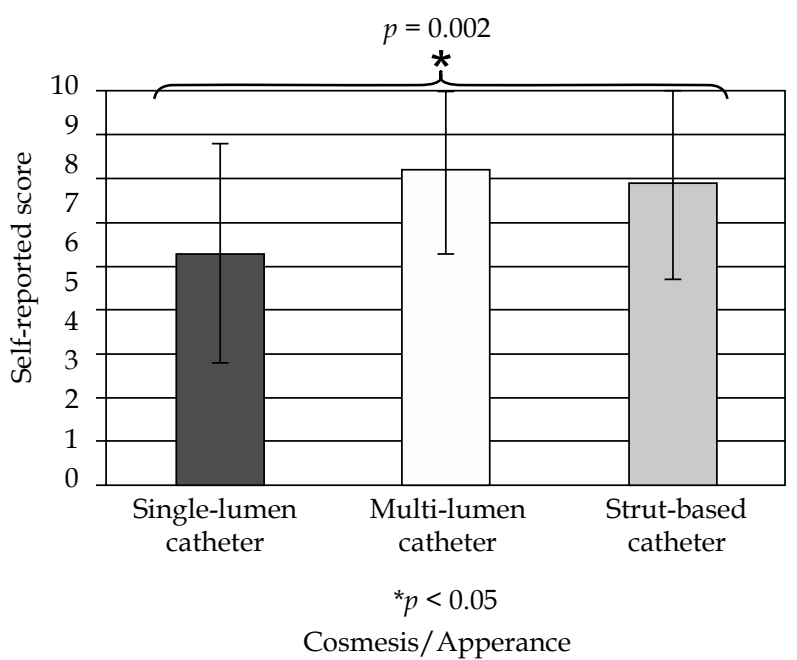

Fig. 3. Influence of balloon type on self-reported cosmesis scores and quality of life measures

sus balloon-based APBI is dependent upon lumpectomy cavity geometry and skin distance, therefore there may be confounding affecting outcomes. If this were the case, however, it would have been expected that the strutbased system perform worse with larger cavities and smaller skin distances.

Despite these limitations, to our knowledge, this is the first non-industry funded study to examine the selfreported cosmesis and quality of life ratings of women treated with single-lumen versus multi-lumen catheter devices and whole breast radiation therapy. Future prospective studies, including larger sample sizes are needed to further characterize the differences between modalities of delivering APBI.

\section{Conclusions}

In conclusion, APBI is a safe and efficacious alternative to WBRT in women with early stage breast cancer following lumpectomy. Multi-lumen catheter APBI may also offer women superior breast cosmesis and decreased toxicity with increased time following their treatment.

\section{Acknowledgements}

This data were presented as a poster at ASTRO's $56^{\text {th }}$ annual meeting in San Francisco, CA, USA in September, 2014.

\section{Disclosure}

Authors report no conflict of interest.

\section{References}

1. Shaitelman S, Lin H, Shen Y et al. Utilization of accelerated partial-breast irradiation (APBI) and guideline concordance: Analysis of the National Cancer Database. J Clin Oncol 2013; 31 (Suppl 26): abstract 54.

2. Vicini $\mathrm{F}$, Winter $\mathrm{K}$, Wong $\mathrm{J}$ et al. Initial efficacy results of RTOG 0319: three-dimensional conformal radiation therapy (3D-CRT) confined to the region of the lumpectomy cavity 
for stage I/II breast carcinoma. Int J Radiat Oncol Biol Phys 2010; 77: 1120-1127.

3. Taghian AG, Kozak KR, Adams J et al. Accelerated partial-breast irradiation (APBI) using protons for patients with early-stage breast cancer: a comparison with 3D conformal photon/electoral based treatment. Int J Radiat Oncol Biol Phys 2005; 63: S8-S9 [Abstract].

4. Livi L, Buonamici FB, Simontacchi G et al. Accelerated partial breast irradiation with IMRT: new technical approach and interim analysis of acute toxicity in a phase III randomized clinical trial. Int J Radiat Oncol Biol Phys 2010; 77: 509-515.

5. Vicini FA, Chen $P$, Wallace $M$ et al. Interim cosmetic results and toxicity using 3D conformal external beam radiotherapy to deliver accelerated partial breast irradiation in patients with early-stage breast cancer treated with breast-conserving therapy. Int J Radiat Oncol Biol Phys 2007; 69: 1124-1130.

6. Antonucci JV, Wallace M, Goldstein NS et al. Differences in patterns of failure in patients treated with accelerated partial breast irradiation versus whole-breast irradiation: a matched-pair analysis with 10-year follow-up. Int J Radiat Oncol Biol Phys 2009; 74: 447-452.

7. Wadasadawala T, Sarin R, Budrukkar A et al. Accelerated partial-breast irradiation vs conventional whole-breast radiotherapy in early breast cancer: a case-control study of disease control, cosmesis, and complications. J Cancer Res Ther 2009; 5: 93-101.

8. Skowronek J, Wawrzyniak-Hojczyk M, Ambrochowicz K. Brachytherapy in accelerated partial breast irradiation (APBI) - review of treatment methods. J Contemp Brachytherapy 2012; 4: 152-164.

9. Weed DW, Edmundson GK, Vicini FA. Accelerated partial breast irradiation: a dosimetric comparison of three different techniques. Brachytherapy 2005; 4: 121-129.

10. Dickler A, Kirk MC, Chu K et al. The Mammosite TM breast brachytherapy applicator: A review of technique and outcomes. Brachytherapy 2005; 4: 130-136.

11. Perera F, Chisela F, Engel M et al. Method of localization and implantation of the lumpectomy cavity for dose rate brachytherapy after conservative surgery for $\mathrm{T} 1$ and $\mathrm{T} 2$ breast cancer. Int J Radiat Oncol Biol Phys 1995; 31: 4959-4966.

12. Kirk M, His WC, Dickler A et al. Surface optimization technique for MAMMOSITE breast Brachytherapy applicator. Int J Radiat Oncol Biol Phys 2005; 62: 366-372.

13. Kini V. Balloon breast brachytherapy - review of current data. Brachytherapy 2005; 4: 181-182.

14. Forszpaniak J. Balloon brachytherapy: how I do it. Nowotwory 2004; 1: 11-14.

15. Keisch M, Arthur DW. Current perspective on the MammoSite radiation therapy system - a balloon breast brachytherapy applicator. Brachytherapy 2005; 4: 177-180.

16. White J. Mammosite and accelerated partial breast irradiation: Rethinking one-size-fits-all breast irradiation after lumpectomy. Brachytherapy 2005; 4: 183-185.

17. Wazer DE, Kaufman S, Cuttino L et al. Accelerated partial breast irradiation: an analysis of variables associated with late toxicity and long-term cosmetic outcomes after high dose rate interstitial brachytherapy. Int I Radiat Oncol Biol Phys 2006; 64: 489-495.

18. De la Rochefordière A, Abner AL, Silver B et al. Are cosmetic results following conservative surgery an radiation therapy for early breast cancer dependent on technique? Int J Radiat Oncol Biol Phys 1992; 23: 925-917.

19. Cella DF, Tulsky DS, Grey G et al. The Functional Assessment of Cancer Therapy (FACT) scale: development and validation of the general measure. J Clin Oncol 1993; 11: 570-579.

20. Ott OJ, Shultz-Wendtland R, Uter $W$ et al. Fat necrosis after conserving surgery and interstitial brachytherapy and/or ex- ternal- beam irradiation in women with breast cancer. Strahlenther Onkol 2005; 181: 638-644.

21. Tanaka E, Yamazaki H, Yoshida K et al. Objective and longitudinal assessment of dermatitis after postoperative accelerated partial breast irradiation using high-dose-rate interstitial brachytherapy in patients with breast cancer treated with breast conserving therapy: reduction of moisture deterioration by APBI. Int J Radiation Oncology Biol Phys 2011; 81: 1098-1104.

22. Flynn C, Mitchell C, Boyea G et al. A comparison of quality of life (QoL) for early stage breast cancer examining various treatment modalities and no chemotherapy. Int J Radiat Oncol Biol Phys 2007; 69: S583-S584 [Abstract].

23. Wadasadawala T, Budrukkar A, Chopra S et al. Quality of life after accelerated partial breast irradiation in early breast cancer: matched pair analysis with protracted whole breast radiotherapy. Clin Oncol (R Coll Radiol) 2009; 21: 668-675.

24. Flynn C, Wallace M, Balasubramaniam M et al. An assessment of quality of life for patients undergoing radiotherapy with whole breast irradiation compared to accelerated partial breast irradiation. Int J Radiat Oncol Biol Phys 2006; 66: S134 [Abstract].

25. Lee K, Quillo A, Dillon D et al. Acute toxicity and early cosmetic outcome in patients treated with multi-lumen balloon brachytherapy with skin spacing $\leq 7$ millimeters. J Contemp Brachytherapy 2012; 4: 8-13.

26. Yashar CM, Scanderbeg D, Quiet CA et al. Outcomes for APBI with strut-based brachytherapy: first 200 accrued patients. ASTRO 2014 Annual Meeting. San Francisco: SAVI Collaborative Research Group, 2014 [Abstract].

27. Rana ZH, Nasr N, Ji H et al. Factors improving skin sparing, rib sparing, and D95 coverage in accelerated partial breast irradiation across five catheter subtypes. ASTRO Annual Meeting. San Francisco, 2014 [Abstract].

28. Elekta. Partial Breast Irradiation With Interstitial Brachytherapy Appears Appropriate For Women Previously Considered Unsuitable. 09 17, 2014. http://article.wn.com/ view/2014/09/17/Partial_Breast_Irradiation_With_Interstitial_Brachytherapy_A_3/(accessed 11 29, 2014).

29. Hologic. MammoSite: How it works. 2014. http://www. mammosite.com/physicians/radiation-therapy/how-itworks.cfm (accessed 1129,2014 ).

30. ITN. IBioLucent Receives FDA Clearance for SAVI Brachytherapy Device 2006. http://www.itnonline.com/article/biolucent-receives-fda-clearance-savi-brachytherapydevice (accessed 11 29, 2014). 